\title{
Pétersen, une gare routière au cour des dynamiques urbaines de Dakar
}

\author{
Mamadou Ndiaye \\ Université Cheikh Anta Diop \\ Dakar, Sénégal \\ Rémy Tremblay \\ Téluq, Université du Québec à Montréal
}

Chaire de recherche du Canada sur les villes du savoir

\section{Introduction}

Le Sénégal, comme de nombreux pays en développement connaît un développement urbain incontrôlé, caractérisé par une insuffisance des offres par rapports aux demandes. Dakar, en tant que capitale constitue une ville macrocéphale qui abrite une grande partie des infrastructures et équipement du pays. A Dakar, la démographie galopante, l'urbanisation massive et l'accroissement des activités industrielles, commerciales et informelles sont à la base de la dégradation de l'espace urbain. Le constat est que très souvent les véhicules stationnent partout et n'importe où, d'où la nécessité de créer des lieux de stationnement et des gares routières indiquées.

La gare routière de Pétersen est située sur l'avenue Faidherbe entre le prolongement de l'Avenue Sénégal et la rue Mangin. Reliée à tous les nœuds de communication en direction de la banlieue, elle joue un rôle non négligeable dans le système de transports urbains de la métropole dakaroise. De par sa position, elle se trouve non loin des $6^{1}$ grandes pénétrantes qui convergent vers le plateau du Nord au Sud. La gare reçoit chaque jour plus de 50000 personnes. Ainsi joue t'elle un rôle non négligeable dans le système de transport

de la capitale. Le mode gestion appliqué à Pétersen est la concession qui diffère des autres modes de gestion. La concession de service public est une convention par laquelle une personne publique (le concédant : ville de Dakar) confie à une personne morale, privée ou parfois publique (le concessionnaire : la SAGES), les soins d'assurer, pendant un temps déterminé la gestion d'un service public (Pétersen). L'importance de la gare routière Pétersen est due à la masse importante de personnes et de bien embarquer et débarquer quotidiennement.

A tout point de vue, Pétersen diffère des autres gares routières du Pays ${ }^{2}$. Aussi bien son mode de construction, d'organisation et de gestion, qui représente un modèle spécifique «formaté from Banque Mondiale » $»^{3}$, constituent des facteurs suscitant l'intérêt des chercheurs. De ce fait, cet espace public très convoité cache des réalités très complexes.

L'importance de la gare routière Pétersen est due à la masse importante de personnes et de bien embarquer et débarquer quotidiennement 
Située au cœur de la ville de Dakar, elle a pour fonction principale d'assurer la mobilité des personnes et des biens entre le centre ville de

\section{Le contexte urbain dakarois}

À cette pression démographique s'ajoute le handicap de la configuration géographique et de la répartition spatiale déséquilibrée des activités économiques et sociales et de l'habitat sur la presqu'île. La ville compte plus des trois quarts des emplois avec une forte concentration dans le Plateau: $27 \%$. La plupart des activités sont concentrées sur le Plateau notamment au voisinage du port, de la zone industrielle et non loin du grand marché métropolitain de Sandaga. Au même moment développent d'autres activités dans les zones de plus en plus éloignées du centre. Il résulte de cette situation et de la dynamique urbaine des migrations quotidiennes qui affectent des populations de plus en plus nombreuses, pour des déplacements de plus en plus longs.

Cette situation est aggravée par la rigidité du réseau de voirie essentiellement composé de cinq grands axes routiers formant des pénétrantes qui convergent vers le Plateau. Le réseau de voirie du Plateau se compose d'une part, d'avenues et de boulevards de 15 à $20 \mathrm{~m}$ de largeur et d'autre part de voiries et de rues de $10 \mathrm{~m}$ de large. On constate aussi une dégradation continue de la circulation liée à l'insuffisante capacité des routes, à l'augmentation des besoins en mobilité et à la faiblesse de l'offre de stationnement. Bastié J et Dézert B, 1980 : « la circulation est le reflet de la structure de l'espace urbain, c'est elle qui indique où sont les pôles de convergences, quels sont les axes communs. ${ }^{4}$

La forte et rapide urbanisation de la capitale par rapport au reste du pays n'est pas sans risques. L'occupation de l'espace urbain engendre beaucoup de difficultés. Dakar, avec $97 \%$ d'urbains a un taux d'urbanisation qui
Dakar et sa banlieue. Le présent article fait une brève présentation de la gare routière.

dépasse très largement la moyenne nationale qui est de $45 \%$. La structure urbaine est caractérisée par une macrocéphalie, c'est-àdire une prépondérance de la part de la capitale dans le pays.

\section{La structure urbaine est caractérisée par une macrocéphalie}

A l'image des grandes villes en développement, Dakar «est une ville instable, dans la mesure où les lieux et leur occupation se transforment rapidement, et où l'appropriation et la désignation des espaces sont sujettes à de très nombreux conflits et à des changements dynamiques $»^{5}$. Beaucoup de phénomènes se produisent avec des répercussions variables tant au niveau des populations qu'au niveau de l'espace urbain qui subit plusieurs sortes de modifications.

En ce qui concerne le contexte socioéconomique, le poids économique de la capitale est considérable. L'écart de croissance économique entre la capitale et les autres régions du pays est énorme et se mesure à tous points de vue. Dakar peut être considérée comme une ville macrocéphale constituant le moteur de l'économie sénégalaise. On y note une forte concentration des activités économiques et une grande centralisation des secteurs : industriel, tertiaire, commercial, administratif, des transports etc. La région de Dakar fournit plus de $55 \%$ du PIB national, les $2 / 3$ des salariés du secteur «moderne », 80 $\%$ des emplois commerciaux et industriels. Le port de Dakar joue un rôle appréciable dans les transactions avec les autres pays car assure environ $80 \%$ du commerce extérieur et 
presque la quasi-totalité des importations. La concentration des instruments du pouvoir politique, économique, exécutif, et le fait que Dakar abrite la plupart des sièges des grandes sociétés du pays font d'elle une ville où tout doit transiter.

La croissance urbaine de Dakar reste aussi marquée par un taux de chômage élevé. Les populations les plus touchées par le chômage sont les jeunes ${ }^{6}$ avec un taux de $41 \%$. Jouant un rôle primordial au niveau du développement urbain, Dakar abrite les services, exploitations et implantations les plus stratégiques du pays. D'après les chiffres fournis par la chambre de commerce de Dakar dans le répertoire des entreprises du Sénégal de 1999, sur 1.105 entreprises déclarées pour l'ensemble du pays, Dakar abrite 991, ce qui représente environs $90 \%$.

\section{La nécessité de la construction de la gare routière Pétersen}

La gare de Pétersen joue un rôle dans le façonnement géographique actuel de Dakar. Elle bouleverse totalement l'aménagement du centre ville. L'espace qu'elle polarise s'élargit sur un très grand rayon et s'étend jusqu'au marché central de Sandaga, l'avenue Malick Sy, le marché de Thilène, le bureau de poste de la Médina etc. Les activités qui se sont développées autour de la gare Pétersen sont nombreuses et n'ont pas toujours de liens directs avec le secteur du transport.

Les flux de circulation et les besoins de stationnement ont augmenté du fait de la possession d'un véhicule et de son utilisation dans toutes sortes de déplacements urbains. Cependant tout déplacement suppose un lieu d'arrivée d'où les nombreux stationnements. Dans le Plateau de Dakar, la fonction de centre ville en fait un lieu de rassemblement, de convivialité, de mixité de population, de richesses culturelles artistiques et historiques, un lieu de confrontation entre offre et demande. C'est également un lieu de forte concentration d'activités commerciales, administratives ludiques et touristiques. Les problèmes de circulation sur le Plateau sont amplifiés par les stationnements anarchiques et par l'inorganisation des modes de transports.

La concentration d'équipements et d'infrastructures structurants dans le centre ville explique la convergence de plusieurs milliers de personnes chaque jour dans le Plateau. En l'absence d'une organisation conséquente de l'espace, des difficultés font jour: encombrements, surutilisation de la voirie urbaine, embouteillages, manque d'espaces de stationnement, etc. Pour palier ces difficultés plusieurs séries de mesures ont été adoptées par les autorités locales, parmi lesquelles la mise en œuvre d'un Plan de Circulation du Plateau (PCP) et des dispositions permettant de mieux organiser les infrastructures existantes. La nécessité de créer une gare routière centrale et polarisatrice s'affirment de plus en plus.

\section{Avant la création de la gare routière de Pétersen, il y'avait une pléthore de petites gares routières dispersées}

En outre, le secteur des transports urbains à Dakar avant la création de la gare routière de Pétersen était caractérisé par une anarchie notable à plusieurs niveaux. Avant la création de la gare routière de Pétersen, il y'avait une pléthore de petites gares routières dispersées dans la ville de Dakar: Esso Port, Tolbiac, Mairie, Lat-Dior, Sham, Thilène et d'autres espaces qui servaient de lieux de stationnement de façon conjoncturelle.

La majeure partie de ces gares routières n'était pas aménagée dans le but de recevoir des véhicules en grand nombre. A titre d'exemple, 
la gare de Esso Port n'avait pas fait l'objet d'aménagements préalables. A force d'y stationner pour prendre des passagers travaillant dans le Port de Dakar, la gare ou le centre ville, le site avait été transformé en gare routière ponctuelle. La remarque est aussi valable pour toutes les unités créées de façon informelle, sans d'équipements. De par leur localisation, elles participaient à l'augmentation des embouteillages dans cette partie de la ville.

Les autorités, dans la tentative de résolution de la question des transports urbains à Dakar ont tenté de regrouper ces petites gares avec la création de la gare routière de Pétersen. La principale raison est d'apporter une réponse à la dispersion notée dans le système de stationnement du Plateau.

La gare routière de Pétersen occupe une place stratégique, du fait de sa situation mais aussi $\mathrm{du}$ rôle primordial qu'elle doit jouer pour participer à l'atténuation du désordre noté dans le stationnement des véhicules. Cette gare constitue un espace très convoité où beaucoup d'activités ont émergé et se développent. Elle participe à l'amélioration de la mobilité des personnes, des biens, de l'information et des services.

\section{Le contexte de la création est aussi marqué par des problèmes de mobilité qui s'accentuaient de jour en jour}

Il existe des problèmes réels de mobilité dans la région de Dakar, du fait de localisation de la plupart des infrastructures sont au centre ville. Une importante partie de la population est obligée de se déplacer quotidiennement pour aller travailler ou pour aller s'éduquer, se soigner, ou s'approvisionner. A cela s'ajoute un parc automobile vétuste et des encombrements résultant de l'état des infrastructures routières.

La mobilité à Dakar est estimée à environ 2,3 déplacements par jour ${ }^{7}$ et par habitants. Il faut, par ailleurs, signaler que le projet de stationnement payant aux heures de grande circulation a permis d'améliorer la circulation dans la zone du plateau. Toutefois, l'offre de transport dans la zone et entre la zone et la région doit être améliorée en quantité et en qualité, pour exploiter au mieux le plan de circulation mis en place en faveur des transports routiers publics. Par ailleurs, la circulation entre le plateau et le reste de la région passe par cinq carrefours stratégiques dont le fonctionnement non satisfaisant entraîne des congestions importantes qui sont parfois systématiques. Cette situation se répercute en particulier sur les carrefours Pétersen et Faidherbe x Lamine Guèye.

\section{La mobilité à Dakar est estimée à environ 2,3 déplacements par jour ${ }^{7}$ et par habitants}

Dans la région de Dakar, on note une diffusion de centres. En effet beaucoup de sites peuvent être considérés de par les activités qui y développées comme centre de la ville de Dakar. L'intensité et l'importance des relations d'échanges, de gestion et de production en témoignent. L'essentielle de ces centres se regroupent dans le Plateau. De ce fait il rassemble tous les pôles de développement: économique, administratif, judiciaire, relationnel etc. On distingue ainsi les pôles (judiciaire, administratif, portuaire et ferroviaire, hospitalier etc.). 


\section{Une kyrielle d'activités concurrence le secteur du transport au niveau de l'occupation de l'espace?}

En entrant à Pétersen on est très surpris, on ne sait plus si c'est une gare routière, un marché ou lieux d'exposition. A côté de l'activité de transport, d'autres types d'activités génératrices de revenus sont menés au niveau de Pétersen. Le taux de fréquentation de la gare et la concentration des activités génératrices de recettes suscitent de nombreuses convoitises. Le commerce est de loin la plus visible et la plus dynamique des activités qui se déroulent au niveau de la gare routière de Pétersen. Elle a connu un regain d'intérêt général surtout avec l'arrivée des commerçants chinois qui se sont implantés dans le plateau avec notamment beaucoup de cantines à l'intérieur et autour de la gare.

Le centre commercial compte 120 boutiques. La plupart sont fermés. Certaines d'entre elles représentent des lieux de stockages de pièces détachées surtout ou d'articles destinés au commerce informel. On remarque l'emprise grandissante des commerçants chinois dans le commerce d'objets divers dans la capitale Sénégalaise. Les marchands ambulants ou «bouticou mbague » ont su profiter des prix bas proposés par les chinois pour assurer leur auto-insertion dans le marché de l'emploi. Ils achètent à des prix très bas des objets qu'ils revendent ensuite. Ils proposent de larges gammes de produits dans divers domaines: vestimentaire, cosmétique, objets artisanaux, meubles et décorations, gadgets, etc.

En ce qui concerne, le secteur de la restauration, elle débute très tôt le matin se poursuit durant la journée. Le prix des repas est à la portée de toutes les bourses (à partir de 50 francs $\mathrm{CFA}$ ). La réparation mécanique constitue aussi une activité très pratiquée.

Divers garages spécialisés dans la mécanique sont présents au niveau de la gare. Le plus souvent l'atelier est situé aux alentours de la gare routière. Le manque d'espaces fait que certains travaux de réparations sont effectués à l'intérieur même de la piste de stationnement. Cependant si la panne ne peut être réparée sur place il est nécessaire alors de déplacer le véhicule vers le garage situé le plus souvent dans l'espace polarisé par Pétersen.

\section{Un espace convoité avec de nombreux enjeux et d'intérêts}

A Pétersen, nous trouvons plusieurs groupes d'acteurs que nous regroupons en deux catégories: Les acteurs physiques (transporteurs, commerçants, restaurateurs, populations, religieux, mendiants et enfants des rues, etc.) et les acteurs moraux (Associations de commerçants, organisations tontinières, associations de riverains, GIE, GPF, etc.). Chaque groupe d'acteurs a sa propre logique, sa conception de l'espace, des intérêts à sauvegarder.

La spécificité de l'environnement propre à la gare met en exergue la collusion de différentes logiques : privées, institutionnelles, socioéconomiques. La multitude des réglementations, les différentes formes de contournement (maraudage) mais surtout l'extension continue de l'emprise foncière des activités du secteur du transport sur les autres espaces publics marchands occasionnent des conflits entre acteurs et dérèglent les principes de la gestion et de l'aménagement du centre ville.

Les modes d'exploitation estampillés Banque Mondiale notamment les systèmes de concession demeurent aujourd'hui au stade embryonnaire et se trouvent, dans le cas de 
Pétersen, en concurrence avec les formes d'endo-gestion $^{8}$ pratiquées par les regroupements de chauffeurs et de coxeurs, du sous-secteur artisanal et celles déployées par les GIE et les GPF exerçant des activités périphériques au transport (gargotières, cantinières, mécaniciens, soudeurs, tôliers...).

L'importance de la gare de Pétersen pour la ville de Dakar se mesure aussi à travers sa participation au développement du secteur informel. Elle est un espace de production dont le caractère informel est devenu majoritaire. Le secteur informel s'impose comme la réalité dominante en matière de production et de création d'emplois. A Dakar «les promesses d'une ville pourvoyeuse d'emplois paraissent aujourd'hui illusoires $\gg 9^{9}$ Les demandes augmentent sans cesse tandis que l'offre ne suit pas.

\section{La concession : un nouveau modèle de gestion des gares routières au Sénégal}

Dans les années 90, dans le cadre du troisième Programme de Développement Urbain financé par la Banque Mondiale, l'ACDI et l'Etat du Sénégal. Le financement des projets prévus dans ce PDU a permis le financement du Plan de Circulation au Plateau (PCP) et de la construction de gares routières dont celle de Pétersen. C'est dans ce cadre que l'îlot Pétersen doit accueillir un site multimodal de transport et un centre commercial tout comme une partie du camp Lat-Dior est transformée en gare routière. En outre, il est prévu de mettre en place des parkings privés ainsi que l'application de mesures dissuasives pour obliger les chauffeurs à stationner dans ces parkings payants. A la fin de l'année 1995, l'îlot Pétersen et l'espace réservé du camp LatDior sont progressivement construits en gares routières et parcs de stationnement modernes aptes à recevoir un nombre important de véhicules.

A la fin des négociations, le mode de gestion retenu est la concession. La Concession de service public est une convention par laquelle une personne publique (le concédant : ville de Dakar) confie à une personne morale, privée ou parfois publique (le concessionnaire: la Sages ${ }^{10}$ ), le soin d'assurer, pendant un temps déterminé la gestion d'un service public (Pétersen). Cette idée de la Banque Mondiale d'éviter que la gestion des nouvelles gares soit l'exclusivité des C.L est reprise par Lombard J et Bruez F, (2000) : «face à la complexité de la situation institutionnelle, accentuée par l'approche des élections locales de décembre 1996, la Banque Mondiale ne veut pas que les communes gèrent directement les gares et commande un rapport sur leur conception et leur fonctionnement pour alimenter la réflexion sur les modalités de gestion de LatDior et de Pétersen ».

Le recours à un opérateur privé suggéré par la BM est devenu nécessaire. Dans les appels d'offre lancés, il est précisé que le cabinet choisi dans le cadre de cette étude va en même temps proposer un type de contrat de concession pour la gestion de gares multimodales (Pétersen, Lat-Dior). C'est la société canadienne Vimax qui a été choisie pour la réalisation du projet.

Le 1er mars 1999 marque la date officielle de démarrage des activités du concessionnaire : la Sages-Vimax. Après une période commune de gestion de 6 mois, la gestion est désormais l'exclusivité de la Sages. Pour mener à bien sa mission la Sages dispose d'un personnel composé de 60 agents dont 17 journaliers. Ces derniers ont pour rôle d'assurer : la sécurité et la surveillance, l'entretien et la réfection du site, la collecte des taxes et le bon fonctionnement de la gare. Le montant collecté 
quotidiennement est de $125000 \mathrm{~F} \mathrm{CFA} \mathrm{et} \mathrm{la}$ part versé au concédant est de 60\% des recettes globales nettes. L'importance de l'intérêt sur le plan financier explique les nombreux conflits notés dans la gestion.

De plus, le mode de gestion par concession permet non seulement aux concessionnaires et à l'Etat de tirer profit de la gestion mais aussi il permet aux «petits acteurs» de tirer leur épingle du jeu et ainsi d'assurer leur survie quotidienne à Pétersen.

Des milliers d'acteurs viennent et travaillent quotidiennement au niveau de la gare routière de Pétersen. C'est lieu de convergence d'intérêts multiples, c'est aussi un espace de tensions et de contractualisation entre chauffeurs, coxeurs, apprentis, acteurs économiques gravitant autour de la gare, usagers, gestionnaires et forces de l'ordre.. La gare reçoit chaque jour plus de 50000 personnes. Ainsi joue t'elle un rôle non négligeable dans le système de transport de la capitale et dans les budgets des ménages. Ceux qui vivent des gares routières sont nombreux. Pour le versement des frais de paiement du personnel travaillant dans ces groupements, il s'effectue quotidiennement : il est de $3500 \mathrm{~F}$ CFA/jour pour les responsables; $2500 \mathrm{~F} \mathrm{CFA}$ par jour pour les chauffeurs et les coxeurs et de $2000 \mathrm{~F} \mathrm{CFA/jour} \mathrm{pour} \mathrm{les} \mathrm{autres.} \mathrm{De}$ nombreux acteurs parviennent à assurer leur survie quotidienne en exerçant des activités qui ne nécessitent pas de grands investissements.

\section{Des milliers d'acteurs viennent et travaillent quotidiennement au niveau de la gare routière de Pétersen}

\section{La nécessité d'une collaboration entre concessionnaire et regroupements de chauffeurs}

La plus importante source de rentrée de fonds pour la Sages étant la collecte des taxes issues des sorties de véhicules. Sachant que cette taxe n'est pas directement prélevée par les agents de la Sages mais par les regroupements de chauffeurs eux-mêmes. Les relations entre la Sages et les regroupements de chauffeurs sont au cour du dispositif original de gestion de la gare de Pétersen. Sans passer par ces regroupements, qui organisent l'exploitation des têtes de ligne et la bonne marche des rotations de cars, il est impossible pour la Sages d'espérer travailler directement avec les chauffeurs qui entrent dans la gare. Actuellement, il n'est pas envisageable de changer ce système tant les groupements de chauffeurs sont puissants et correspondent à une logique dominante de structuration du secteur.

En effet, pour la Sages, la principale source de rentrée d'argent est la taxe perçue sur chaque départ de véhicule de transport en commun. Les différents regroupements de chauffeurs présents dans la gare gèrent chacune des têtes de ligne (cf. carte sur les trajets des différents regroupements de chauffeurs) sur lesquelles s'inscrivent les cars dès leur entrée dans la gare. Ces cars, en sortant de la gare routière, paient une taxe de 300 à $400^{11} \mathrm{~F}$ CFA. Une partie de cette somme est versée à la Sages (100 F CFA par départ) tandis que le reste revient au groupement de chauffeurs qui l'utile pour son propre compte. Cette situation est décriée par le gérant de la gare pour la Sages pour qui : «la part versée par les groupements de chauffeurs lors de chaque départ est trop minime, elle doit être revue à la hausse ».

Pour le contrôle du nombre de départ, un agent de la Sages est présent devant chaque sortie et distribue des tickets aux chauffeurs tandis ces derniers versent directement l'argent aux collecteurs de taxe du groupement qui 
organisent la tête de ligne et dont ils sont le plus souvent affiliés. Autant de sources de conflits qui non résolues peuvent mener vers des conflits incontrôlables.

\section{Les autres acteurs intervenants dans la gestion de la gare routière Pétersen}

La gestion de la gare routière Pétersen n'est pas exclusivement dévolue à la Sages, il existe d'autres acteurs qui jouent des rôles appréciables et parviennent à assurer leur survie. En effet, il existe d'autres intervenants dans la gestion que cela soit de façon directe ou indirecte. Hormis les acteurs du transport, d'autres acteurs interviennent: les usagers, les populations, les acteurs du secteur commercial et de secteur du transport. Parmi ceux-ci, nous retiendront: les populations (les riverains c'est-à-dire ceux habitant l'espace polarisé par de la gare routière et les usagers ou passager qui viennent à la gare pour prendre le car), les acteurs du secteur des transports (transporteurs et tous ceux qui travaillent dans des activités dépendantes du secteur), les acteurs du secteur commercial (qui sont le plus souvent des «commerçants informel », les municipalités (CADP et Ville de Dakar). Pour que la gestion de Pétersen soit parfaite, il est nécessaire d'impliquer tous ces acteurs dans les différentes étapes du processus de développement.

Il est vrai que la SAGES, Association, de Financement des Transports Urbain, les chauffeurs et les regroupement de chauffeurs de Ndiaga Ndiaye et de Tata sont les plus visibles et les plus présents, mais il existe aussi d'autres tels que les coxeurs qui organisent et gère les lignes, laveurs qui s'occupent du nettoiement des cars, les organisations d'opérateurs qui peuvent avoir plusieurs buts : économique, social, religieux etc., et les acteurs étatiques représentés surtout par les collectivités locales (Mairie de Dakar et commune d'arrondissement de Dakar Plateau) qui perçoivent des taxes municipales, les préfectures qui prélèvent les droits de stationnement et les services de police qui contrôlent le respect des normes. Il existe aussi d'autres acteurs tels que les sociétés d'assurance, les mécaniciens, les financiers (investisseurs, banques etc.).

Au niveau de la gare, on note un important développement de l'activité de restauration qui occupe de plus en plus d'espace. Ces vendeurs occupent le prolongement de l'Avenue Sénégal à l'intérieur de la gare. Ils sont rangés sur une colonne en ligne continue et semble être bien organisés avec leurs étales caractérisées par leur précarité. Le prix des repas est à la portée de toutes les bourses (à partir de 50 francs CFA).

Cette activité qui débute très tôt le matin se poursuit durant la journée. Le soir elle perd de son ampleur jusqu'à devenir quasi-inexistante à certaines heures de la soirée. Les raisons qui expliquent ce phénomène sont multiples. Pétersen est plus un lieu de transit qu'un lieu de d'arrêt pour les passagers, il n'existe pas de moyens de transport à destination de la gare. La localisation de la gare, dans un lieu très fréquenté en fait un des espaces les plus fortement convoités du centre ville.

Le commerce informel est, de loin, la plus visible et la plus dynamique des activités qui se déroulent au niveau de la gare routière Pétersen. Il a connu un regain d'intérêt général surtout avec l'arrivée des commerçants chinois qui se sont implantés dans le plateau avec notamment beaucoup de cantines à l'intérieur et autour de la gare de Pétersen. Il existe aussi un centre commercial avec des boutiques dont la plupart sont fermés. Certaines d'entre elles représentent des lieux de stockages de pièces détachées surtout ou d'articles destinés au commerce informel. Les marchands ambulants 
ou «bouticou mbague» ont su profiter des prix bas proposés par les chinois pour assurer leur auto-insertion dans le marché de l'emploi. On note aussi la présence d'autres acteurs informels exemple: des vendeurs d'eau fraîche, de glace, de boissons, des vendeurs de thé, café, lait, des vendeurs de journaux, des cireurs de chaussures, des laveurs de voiture, des handicapés, des guérisseurs et des

\section{Une forte dynamique associative}

Lieu de convergence d'intérêts multiples, il est un espace de tensions et de contractualisation entre chauffeurs, coxeurs, apprentis, acteurs économiques gravitant autour de la gare, usagers, gestionnaires et forces de l'ordre. Certains de ces acteurs ont vu la nécessité de s'associer en formant soit des groupements, des mutuelles, des associations etc. l'intérêt des regroupements se ressent surtout dans les moments de détresse ou en cas de problèmes ou de déguerpissement.

Cependant, le constat est que la dynamique associative est assez forte ou se fait de façon informelle. En effet, il n'existe que peu d'associations reconnues avec des textes réglementaires, le plus souvent les populations se regroupement par affinité ou en fonction d'intérêts communs. Les acteurs sont plus dans des logiques de concurrence et de rivalité que d'organisation. Pour la prise en charge des questions sociales il est nécessaire de réaliser un important travail de renforcement des capacités organisation-nelles et associatives.

Sur le plan syndical, la dynamique associative est assez forte à Pétersen. Les transporteurs se regroupement le plus souvent en syndicat pour défendre la profession En outre, certains de ces acteurs ont vu la nécessité de s'associer en formant soit des groupements, des mutuelles, des associations etc. l'intérêt des regroupements se ressent surtout dans les moments de détresse ou en cas de problèmes vendeurs de produits médicaux traditionnels et pharmaceutiques etc.

Les acteurs du secteur informel et ceux du transport du fait des intérêts économiques en jeu, se resserrent les coudes pour contrecarrer les plans de l'institution étatique et du concessionnaire sans violence.

ou de déguerpissement. On note l'existence de 4 groupements (Le groupement de Tolbiac/Mairie, Le groupement de Esso/Port, Le groupement des Chauffeurs de Taxis, Le groupement de Faidherbe/Pétersen) de chauffeurs dont les membres peuvent aussi souscrire au S.N.T.T.R.S/C.N.T.S et la mutuelle de santé avec actuellement 200 membres. L'agence de financement des transports urbains qui organisent les Tatas offre aussi un certain nombre d'opportunités à ses membres. Les acteurs sont affiliés aux grands syndicats de transport: CNTS, CNTS FC, etc. Il existe une véritable chaine de solidarité et d'entraide. A titre d'exemple, le travail des apprentis chauffeurs est à comprendre en relation avec les charges et les obligations quotidiennes qui incombent aux chauffeurs. Les relations entre les apprentis et leurs employeurs (les chauffeurs) apparaissent dans la réalité à travers un système de collaboration. Chaque apprenti va suivre les ordres et les directives de son chauffeur afin de faciliter la communication en leur sein.

\section{De façon notoire, les acteurs du secteur des transports participent et négocient de façon collective les questions d'intérêt général}

Les acteurs de la gare routière Pétersen contribue aussi aux prises de décision en matière de définition de politique publique en 
matière de transport. Ils sont affiliés à des syndicats et revendiquent l'amélioration de leur condition de travail et d'existence. De plus en plus les syndicats de transports sont impliqués dans les sphères de prise de décision concernant le secteur des transports. La Sages en tant que concession et gestionnaire du stationnement payant dans le centre ville participe et influence les décisions concernant Pétersen au niveau externe.

\section{Conclusion}

Les effets induits par la construction de la gare routière de Pétersen sur le remodelage actuel de la ville de Dakar doivent être mis en exergue. Dans le contexte d'une ville caractérisée par des problèmes liés à la mobilité urbaine, la construction d'équipements destinés à recevoir des acteurs $\mathrm{du}$ transport participe à une meilleure organisation du système des transports. La construction d'équipements et d'infrastructures nouvelles pour la réorganisation de ce secteur et l'accueil des unités de transports constitue des débuts de réponses dans la gestion du secteur. La construction des gares routières telles que: Pétersen et Lat-Dior est à inscrire dans cette dynamique.
De façon notoire, les acteurs du secteur des transports participent et négocient de façon collective les questions d'intérêt général. Au l'intérieur de la gare, la collaboration entre la Sages et les regroupements de chauffeurs permet d'éviter des querelles entre gestionnaire et acteurs et contribue à l'amélioration de la gestion du secteur. L'implication des acteurs lors de prises de décision permet à la Sages montre qu'il existe une gestion concertée et approprié par les acteurs du transport.

La gare routière de Pétersen occupe une place stratégique, du fait de sa situation mais aussi du rôle primordial qu'elle doit jouer pour participer à l'atténuation du désordre noté dans le stationnement des véhicules. Cette gare constitue un espace très convoité où beaucoup d'activités ont émergé et se développent. Elle participe à l'amélioration de la mobilité des personnes, des biens, de l'information et des services.

L'occupation de l'espace montre la diversité des intervenants avec des intérêts divergents parfois conflictuels. Le fonctionnement de cet espace est très complexe et varie en fonction des nombreux enjeux qu'il suscite.

\section{Notes et références}

1 Ces axes de pénétrants au Plateau sont : Corniche Ouest/Peytavin; Blaise Diagne/Jean Jaurès ; Allées Papa Guèye Fall/Faidherbe/Pinet Laprade ${ }^{1}$; Autoroute/Félix Eboué/Pétersen ; Autoroute/Tolbiac/Faidherbe/Pinet Laprade ; et Route de Rufisque/Félix Eboué/Pétersen ou Faidherbe

2 Excepté la gare routière Lat Dior avec laquelle il existe de multiples similitudes

Le modèle de gestion est la concession

4 Bastié J et Dézert B, 1980, l'espace urbain, édition Masson, Paris, New York, Milan Barcelone, 381 p

5 Leimdorfer François, Enjeux et imaginaires de l'espace public à Abidjan (article extrait de Politique africaine $\mathrm{n}^{\circ} 11$; Paris ; Khartala pp 51-75

6 La population dakaroise est majoritairement jeune avec environs 65\% du total, (DPS, 2004)

7 République du Sénégal, ministères des transports terrestres, cellules de coordination du programme sectoriel des transports, « deuxième programme sectoriel des transports (PST II) », Juillet 1988, sous la coordination de El hadji Ibrahima Fall 
8 Les différentes formes de gestion qui sont pratiquées au niveau de la gare de Pétersen incarnés le plus souvent par les coxeurs, les regroupements de chauffeurs, les autres acteurs....

9 Kaspar Wyss, Mamadou Ndiaye, Ndiékhor et Pierre Jacolin ; 2000 ; « villes en sursis au Sahel » ; Harmattan ; Institut tropical Suisse et Enda Graf Sahel ; 280p

10 SAGES : Société Africaine de Gestion et de Stationnement

11 Paiement variant en fonction du nombre de place existant dans le car seul les cars de plus de 42 places paient 400 F CFA

\section{Bibliographie}

1. BASTIE J et DEZERT B (1980), l'espace urbain, édition Masson, Paris, New York, Milan Barcelone, $381 \mathrm{p}$

2. Direction de la Prévision et de la Statistique (DPS), (janvier 2004), projections de population du Sénégal issues du recensement de 2002,

3. GODARD Xavier (2000), Difficultés d'adaptation des approches du transport pour les villes en développement. Analyse critique d'études des bureaux internationaux à Dakar ; in les cahiers scientifiques du transport $\mathrm{N}^{\circ} 39$

4. Info 7, 21 (mai 2002), l'invasion automobile,

5. KASPAR Wyss, Mamadou Ndiaye, Ndiékhor et Pierre Jacolin (2000) ; « villes en sursis au Sahel » ; Harmattan ; Institut tropical Suisse et Enda Graf Sahel ; 280p

6. LOMBARD J et BRUEZ F (2002), la Sages à Dakar : un opérateur privé pour une gestion trouble des gares de Pétersen et Lat-Dior, $13 \mathrm{p}$

7. Marchés hebdomadaires (Mardi 21 septembre 2004), «Populations riveraines et commerçants : Une cohabitation en dents de scie »

8. Marchés hebdomadaires, (Mardi 21 septembre 2004), Les espaces de la discorde - auteur : Joseph Birame Sène

9. NDIAYE El Hadji Mamadou (2005), «Dynamiques, changements et gestion des espaces publics en milieu urbain : la gare routière Pétersen de Dakar », mémoire de maîtrise UGB, $152 \mathrm{p}$

10. NDIAYE El Hadji Mamadou (2006), « gestion urbaine et espaces publics : enjeux et conflits d'intérêts dans la gare routière Pétersen de Dakar », mémoire de DEA UCAD, $84 \mathrm{p}$

11. Ndione E. S. (1987), Dynamique d'une société en grappe: un cas, Dakar. Dakar, ENDA-Editions

12. NIANG A (2000), «les associations en milieu urbain dakarois : classification et capacité développante », In Africa development, Vol XXVn¹-2, pp 99-159

13. NOIN D et Thumerelle TP,(1995), 1 'étude géographique des populations, édition Masson, $124 \mathrm{p}$

14. O`DEYE M, (1985), « les associations en villes africaines Dakar-Brazzaville » édition Harmattan Ville et Entreprises, $125 \mathrm{p}$

15. République du Sénégal, (CETUD), (avril 2001), 'bilan et perspectives du conseil exécutif des transports urbains de Dakar', 11 ième réunion du comité d'orientation, SSATP - Mobilité urbaine, Accra, Ghana,

16. République du Sénégal, ministères des transports terrestres, cellules de coordination du programme sectoriel des transports, « deuxième programme sectoriel des transports (PST II) », (Juillet 1988), sous la coordination de El Hadji Ibrahima Fall

17. Sud Quotidien, (15 octobre 2004), «Dakar, 14 oct - Un homme d'une trentaine d'années a trouvé la mort, jeudi soir à la gare routière de "Pétersen"

18. Wal fadjri du 03.12.2004 «Mobilité urbaine : Les occupants de la voie publique seront déguerpis » auteur : Yate Nara Ndoye (stagiaire)

19. THOMAS François, (2001), «l'espace public, un concept moribond ou en expansion » in Géocarrefour vol 76, CNRS-UMR 5600, Environnement-ville société pp 75-84

20.ZOUHOULA BI Marie, «la gare interurbaine au cœur des conflits entre la mairie d'Adjamé et la ville d'Abidjan » pp 1-6 


\section{Publicité}

\section{CRISE}


Notes 
Notes 
Notes 
Notes 
Formulaire d'abonnement 
